\title{
A Novel Data Aggregation Scheme for Wireless Sensor Networks
}

\author{
Syed Gul Shah ${ }^{1}$, Atiq Ahmed ${ }^{2}$, Ihsan Ullah ${ }^{3}$, Waheed Noor ${ }^{4}$ \\ Department of Computer Science \& Information Technology, \\ University of Balochistan, Quetta (87300)
}

\begin{abstract}
Wireless sensor networks (WSN) consist of diverse and minute sensor nodes which are widely employed in different applications, for example, atmosphere monitoring, search and rescue activities, disaster management, untamed life checking and so on. A WSN which is an accumulation of clusters and information exchange occurs with the assistance of cluster head (CH). A lot of sensor nodes' energy is utilized in procedures like detection, information exchange and making clusters using various protocols. In a cluster based WSN, it is profitable to segregate the tasks performed by cluster heads as a fair amount of energy could be conserved. Following this, we propose a solution to include a supplementary node that is named as a 'super node' alongside cluster head in a cluster based WSN in this work. This node is in-charge of all the clusters in a WSN and takes care of the entire cluster's energy information. It manages the cluster heads from their creation to the end. All the clusters in the network send their respective information to this node that eliminates redundant information and forwards the aggregated information towards the sink. This not only saves the $\mathrm{CH}$ energy but also conserves individual cluster node's energy by proper monitoring the energy levels. This mechanism enhances the lifetime of the network by minimizing the number of communications between nodes and the sink. In order to evaluate the performance of our proposed mechanism, we use various parameters like packet delay, communication overhead and energy consumption that show the optimality of our approach.
\end{abstract}

Keywords-Wireless Sensor Networks; energy consumption; energy-aware routing; clustering; data aggregation

\section{INTRODUCTION}

Wireless technology is an essential for today's world because it assists us in modern society, like communication, war, health, disasters management and different other scientific fields. In wireless technology, wireless sensor network (WSN) is recognized as group of spatially scattered minute devices called as sensor nodes that are helpful to detect any event in the environment. These sensor nodes cooperatively work to sense certain phenomenon in any deployed area of interest and send the detected data to the sink also considered as base station (BS).

Each sensor node consists of a radio transceiver with an antenna, an electronic circuit linked with the sensors, a microcontroller, and an energy source, commonly a battery or a fixed form of energy source [1]. Sensor nodes are dispersed over wide space and send gathered data to one or many central nodes called as sink. With incorporation of sensing information, wireless communication, and calculation, the sensor nodes are capable to sense physical information, process detected information, and send this detected information to the sink. Then, the sink probes the data received from sensor nodes. All this procedure consumes a lot of energy which is an important challenge to tackle in WSNs.

WSN is a vigorous innovation which can be used in numerous application domains, however, the present protocols of WSN are not capable enough to manage applications of high mobility sensor nodes of WSN [2]. To optimize the lifespan of sensor node, protocols must be energy proficient in order to decrease energy utilization in each layer which are fundamentally (1) the physical layer, which is used for power consumption control, (2) MAC layer for retransmission control and (3) system layer which is used for routing procedures. Energy consumption in WSN is considered as one of the key challenges to be dealt with.

For data aggregation, the sensors within the network are divided into groups, this grouping of nodes in clusters is named as clustering. Clusters (as shown in Fig. 1) consist of sensor nodes, aggregator nodes and also the querier. Sensor nodes sense the info from surroundings that is sent to the aggregator nodes where it is aggregated. Aggregated knowledge is sent to the querier node that generates the query. The main purpose of the data aggregation is to make sensor networks energy efficient so the network life time may be enhanced as most of the energy is consumed in data communication from node-tonode or to sink.

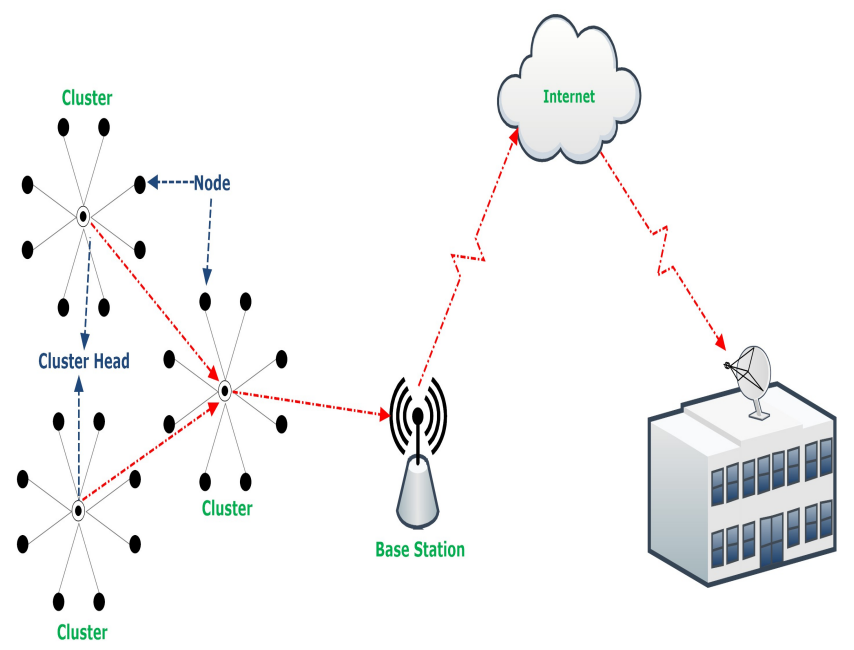

Fig. 1. A Typical Wireless Sensor Network with clusters

It is a method of grouping and aggregating the substantive information by eliminating redundancy. As delineated, it is a vital procedure for energy saving to boost the lifespan of a network as they have limited process power, limited memory 
and battery. This method is employed to resolve the overlap issues in data centric routing as well. It tries to aggregate knowledge from the sensors in an energy efficient manner having minimum latency that is largely the delay concerned in data transmission, routing and aggregation.

In this work, we propose a new data aggregation strategy that aims at reducing the energy consumption with the help of an additional node which is introduced before the sink in order to reduce the number of transmissions towards the sink. We have organized this work as follows. Next section gives an insight into the existing clustering and aggregation methods. We discuss their pros and cons. In Section III, our propose data aggregation method is discussed followed by the evaluation study of our scheme in Section IV. Finally, we conclude this work with some future directions.

\section{RELATED WORK}

In this section, we present some of the schemes that aim at making the WSNs energy efficient. The state-of-the-art protocol for sensor nodes is Low-Energy Adaptive Clustering Hierarchy (LEACH) [3]. By using LEACH, we can achieve cluster-based energy efficient routing. In $\mathrm{LEACH}$, a cluster head $(\mathrm{CH})$ is chosen in every cluster that allows media accessibility with application-oriented data collection to obtain better performance by removing data redundancies and minimizing the data packets which will be sent by cluster heads to their base station. In LEACH all nodes are primaries; at any given time, a few primaries elected as cluster heads, and others are standard nodes. Every standard node inside each cluster sends data to its own specific cluster head intermittently.

Ya et al. [4] have discussed various protocols and LEACH based protocols are assumed to be very efficient in terms of energy utilization. It can sustain two-way transmission since LEACH is designed for single-hop network. Authors also asserted that LEACH cannot ensure $100 \%$ connectivity amongst normal and head nodes because of which, it is not reliable enough. Therefore, Li et al. [5] have put forwarded improvements in LEACH protocol. In this approach, selection of $\mathrm{CH}$ is dependent upon the residual energy of nodes in clusters. Using this method, selection of head nodes process can be balanced as well as robustness and lifetime of the network can be enhanced. Simulation results depict that this algorithm performs better than LEACH with respect to number of alive nodes, consumption of energy and data transmission.

Authors in [6] discussed and compare two diverse secondlevel hierarchical protocols; directed diffusion LEACH (DDLEACH) and two-level LEACH (TL-LEACH). They asserted that the existing clustering schemes are more energy efficient. DD-LEACH protocols devours less energy by the passage of time, however, it gives some expanded delay in the network. TL-LEACH is a more suitable protocol in situations where the event parameter being detected is every-changing. TL-LEACH can be transformed to last longer, if it is factually discovered that sensor nodes at the top level hierarchy are devouring more energy and are furnished with some energy source.

Following the same path, new routing algorithms namely Hierarchical LEACH (H-LEACH) and Hierarchical LEACHDT (H-LEACH-DT) are proposed [7] which are hierarchical extensions of LEACH. By adding these extensions, LEACH protocol would be scalable for large scale WSN and network lifetime could also be optimized. Result show that hierarchical routing protocols work better than LEACH and LEACH-DT. Authors suggested that greater performance of large-scale WSN can be achieved by enhancing the number of progressive levels which are employed in hierarchical implementations of LEACH.

Many other protocols are also employed by the research community to cope with the energy related issues in WSNs. In [8], efficient energy cluster-based routing Protocol (EECRP) is proposed for improving energy utilization by uneven clustering and choosing an improved cluster head with swarm optimization algorithm. This also helps in solving the blind nodes and hot spot problems individually, and by using EECRP the lifespan of the network is considerably improved. However, impact of WSN parameters has not been optimized and some considerable work needs to be done for accurate results. Authors in [9] have combined LEACH and PEGASIS ${ }^{1}$ to form EBLP $^{2}$ and tried to resolve many issues with these protocols. Simulation results show that by utilizing EBLP every node of the system use more adjusted energy utilization which enhances the life cycle of WSN.

CIVIC $^{3}$ [2] is a protocol specifically intended for mobile ad-hoc networks (MANETs). It is based on energy-aware and one-hop broadcast routing mechanism. CIVIC has outperform in the perspective of delay in the data and energy consumption and packet lost rate. Results show that CIVIC can fulfill the requirements of MANETs as well as of WSN. CIVIC is constructed on two key features i.e., energy aware routing and directional broadcast which allows to manage the high mobility of networks and energy based routing intended for optimization of the nodes lifespan of WSN.

Energy hole issue in LEACH has been resolved by utilizing distributed clustering scheme [10] based on energy level of neighboring nodes and sinks, etc. In this technique, authors have proposed to change the cluster head dynamically through likelihood, by considering contrast amongst the nodes' remaining energy and the average energy of its one-hop neighbor. After selection of the cluster head, a bi-election system is executed to discover the group heads in regions and enhance the distribution of $\mathrm{CHs}$ by compensation. Evaluations depict that the recommended clustering algorithm extends the lifespan of WSN and balance the energy utilization among sensor nodes in comparison to LEACH protocol.

Apart from these protocol solution, there is an extensive amount of literature that aims at energy conservation in WSNs. Extending network lifetime is a significant objective for optimization in sensor based networks. Biazi et al. [11] suggested a new mechanism that is based on Time Division Multiple Access (TDMA) by which the energy usage can be minimized. By using this method, WSN energy consumption is reduced up to $17 \%$ in the poorest case and upto $52 \%$ in ideal case. This method moderates the energy consumption of the network and therefore, ameliorates its lifespan. In [12], cost function based solution is proposed for energy-aware cost based routing algorithms. Cost limits can layout the changes

\footnotetext{
${ }^{1}$ Power-Efficient Gathering in Sensor Information Systems

${ }^{2}$ Energy Balanced LEACH and PEGASIS

${ }^{3}$ Communication Inter-Véhicules Intéligente et Coopérative
} 
in residual energy in a node to the big transformation in the function value by furnishing an adjusted and proficient energy use among nodes. Results reveal that the proposed algorithm has better performance than the traditional routing algorithms.

By looking at the above literature, it can easily be observed that energy efficiency is still an area where more work needs to be done. This not only affects the lifetime of the sensor network but also reliability of information. Therefore, we propose a novel algorithm in the next section that not only increases the lifespan of network but also optimizes the communication overhead and energy consumption.

\section{PRoposed Protocol}

This section presents and discusses in detail the functionality of our proposed aggregation algorithm (Fig. 2). For reducing the number of frequent communications between cluster heads and sink, we have introduced a supplementary node that gathers data from cluster heads and transmits that to the sink or other sensor nodes in the network when required. We call it a 'super node' in this work.

Super node is responsible for announcements of events, namely, cluster head election and supervision of overall clusters for energy related issues. Whereas, cluster head only receives the sensor's gathered data about the task they are assigned and it forwards data towards the sink or BS. Frequent communication about the residual energy is done between cluster-heads and super node. When the super node observes that the cluster head has exhausted $25 \%$ of its initial energy, it executes election process. During the electoral process, cluster head continues its work.

Our proposed algorithm has five phases in a single round and the rounds are dependent upon the available nodes in the clusters and their residual energy as well as their sensing environment and tasks. This algorithm is depicted in Fig. 2 and it works as follows. During the election phase, cluster head sends the notifications to the cluster nodes about the probability of becoming a cluster head based on equation (1) [5].

$$
T(n)= \begin{cases}\frac{p}{1-p\left(\operatorname{rmod} \frac{1}{p}\right)} & n \in G(\text { set of nodes }) \\ 0 & \text { Otherwise }\end{cases}
$$

Suppose $x$ be an arbitrary number somewhere in the range of $0-1$ where $n$ is any given node, $p$ is the probability, $r$ is the current round, $G$ is the set of nodes that were not $\mathrm{CHs}$ in the past rounds and $T(n)$ is the predefined threshold. The node moves towards becoming a $\mathrm{CH}$ during the current round if the number is within the range of $T(n)$. After becoming a cluster head, the same node can become cluster head again because in network any node can have less energy than this node. This helps for the optimization of WSN Lifespan.

After this procedure, cluster head advertises its status as a cluster head to all available nodes in the cluster. After this all nodes acknowledge the message and send the join request to the cluster head. Then, cluster Head sends unicast advertisement selecting the $1^{\text {st }}$ respondent of the advertisement as a super node. This selected super node is responsible for selecting cluster head in the next rounds based on the energy matrix (which is maintained by super node). This message will also have the detail of super node (or other $\mathrm{CH}$ ) through which the cluster head intends to send the information towards base station or the address of base station.

Super node sends the broadcast message to all nodes in the networks and as a response all nodes in the specific cluster send their energy messages comprising of their residual and initial energies and active time of the sensor. From this information, super node becomes capable to assess the priority among the nodes based on their energy consumption and active time. Nodes in the clusters send their detected information messages to their respective cluster heads from where, energy updates are forwarded to the super node. Cluster heads also send their energy details to the super node. On the observation of $25 \%$ dissipation of energy from any $\mathrm{CH}$, the super node replaces $\mathrm{CH}$ to the node with maximum energy in the energy matrix and updates the information of previous cluster head in the energy matrix. All normal nodes save their energy by scheduling their transmission and they turn off their transmitter when in idle position, as well as turn it on once they need to transmit any update to the $\mathrm{CH}$ or to the super node.

In the next phase, super node makes a transmission plan based on TDMA [13] for all available sensor nodes in the cluster. TDMA plan depends on the aggregated number of available sensor nodes in the cluster. Every node communicates the information just in the allotted time schedule except in any emergency like when the sensor detects any event or in any situation where major change in the residual energy of the senor appears due to temperature, sensing or processing. After this phase, all nodes in a cluster sends data to $\mathrm{CH}$ and energy messages to the super node. The cluster head sends and receives data to and from BS directly or through some other $\mathrm{CH}$. Sensors in a cluster tend to communicate only with single hop transmission.

On receiving any data, cluster head sends acknowledgment message to the nodes, if in any situation the acknowledgment is not received to a node, after waiting for a specified time period which depends upon the network size, node transmits data again. This triggers the $\mathrm{CH}$ unavailable event at the super node level which is the final phase in aggregation. Super node sends forth data to $\mathrm{BS}$ directly or through any $\mathrm{CH}$ of neighboring cluster. It checks the energy matrix for the cluster head last response, if the cluster head energy entry in the energy matrix is available as normal. Super node broadcasts cluster head alive message to all nodes in the cluster. Otherwise, super node assigns cluster head responsibilities to the node with maximum energy in the energy table and broadcast information about the change of $\mathrm{CH}$ and update the information of previous cluster head in its energy table.

\section{RESUlTS AND Discussion}

In this section, we present the evaluation and comparison of our proposed data aggregation strategy. We have used Matlab [14] which is a widely employed tool in wireless sensor networks for performance measurements. We opted to compare with the existing implementations of LEACH and LEACH-C protocols due to their mechanism pertinence with our proposed approach in terms of lifetime, energy consumption, overhead and packet latency. We have used the following parameters (Table I) for simulation. 


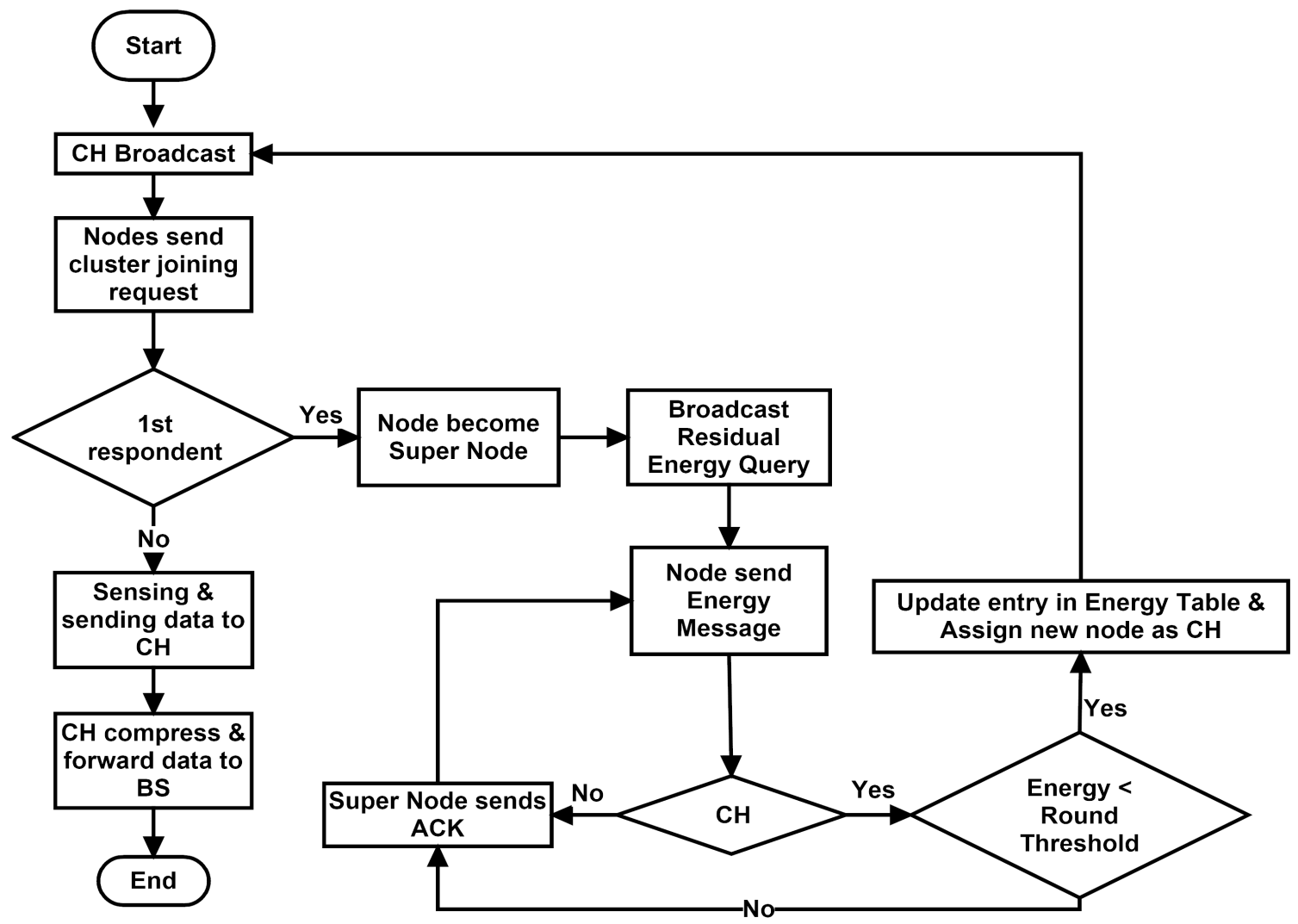

Fig. 2. Flowchart of the Algorithm

TABLE I. Simulation Parameters

\begin{tabular}{|l|l|}
\hline Parameter & Value \\
\hline Simulation time & $60 \mathrm{Mins}$ \\
\hline Area of simulation & $1000 \mathrm{~m} \times 1000 \mathrm{~m}$ \\
\hline No. of Sensor nodes & Variable (Min 50 - Max 100) \\
\hline No. of Simulations & 100 \\
\hline Maximum Packets Sent & 100 \\
\hline Probability to become a super node & 0.2 \\
\hline Node's Initial Energy & $0.5 \mathrm{~J}$ \\
\hline Energy (Transmitter) & $30 \times 10^{-8} \mathrm{~J}$ \\
\hline Energy (Receiver) & $30 \times 10^{-8} \mathrm{~J}$ \\
\hline Energy for data aggregation & $4.5 \times 10^{-5} \mathrm{~J}$ \\
\hline Maximum rounds & 5000 \\
\hline Operating Frequency & $30 \mathrm{kHz}$ \\
\hline
\end{tabular}

Fig. 3 reflects the performance of our proposed approach. It can observed that lifespan of the nodes in our approach is better than LEACH and LEACH-C and deployed nodes remain alive for a longer period. This is due to the distributed clustering and optimal selection of the $\mathrm{CHs}$ and super node. As soon as any node starts excessive energy dissipation being a $\mathrm{CH}$ and its energy level reaches the predefined threshold, it is replaced by an energy efficient node in order to keep the nodes alive for a longer period. LEACH and LEACH-C do not follow this sort of discriminative behavior during $\mathrm{CH}$ selection procedure.

Fig. 4 shows the results regarding the energy consumption

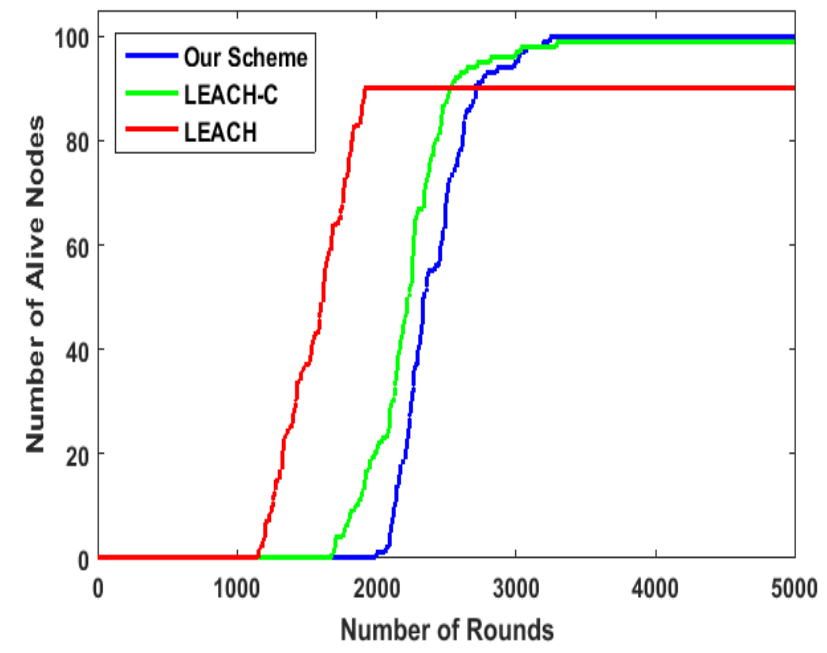

Fig. 3. Number of Alive Nodes

of sensor nodes for a maximum number of rounds. Energy consumption highly depends upon the placement of base station. Farther the BS from the sensing nodes, more is the amount of energy consumed by the nodes for data transmission. In our case, nodes consume very less energy as they transmit their data to respective $\mathrm{CH}$ which hands over this data to super node. Super node then does all the tasks of data transmission towards the sink. This saves a large amount of energy of contributing 
nodes and making our scheme better than the two others.

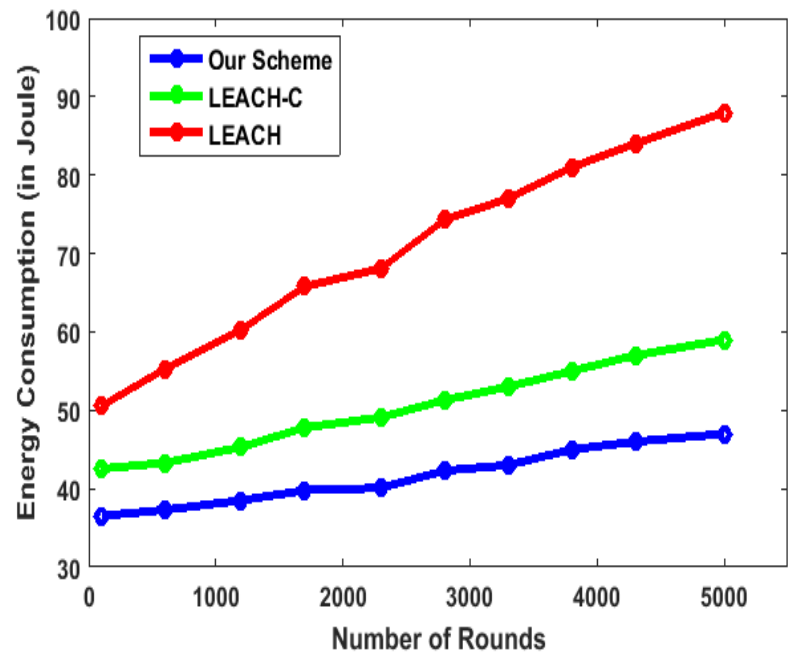

Fig. 4. Energy consumed during maximum runs

Packet delay can be observed in Fig. 5. Our scheme performs marginally better than LEACH and LEACH-C. These algorithms form clusters unevenly and undesirably because cluster head formation is done by the base station anytime. Therefore, at any moment when nodes desire data transmission but network BS decide to create or break any cluster at that time then there will be a lot of latency. However, in our proposed scheme data transmission is done by the super node which is not dependent upon cluster creation or destruction thus, providing marginally low packet latency than LEACH-C and LEACH. However, more work needs to be done on this aspect of our proposed algorithm to get some optimal results.

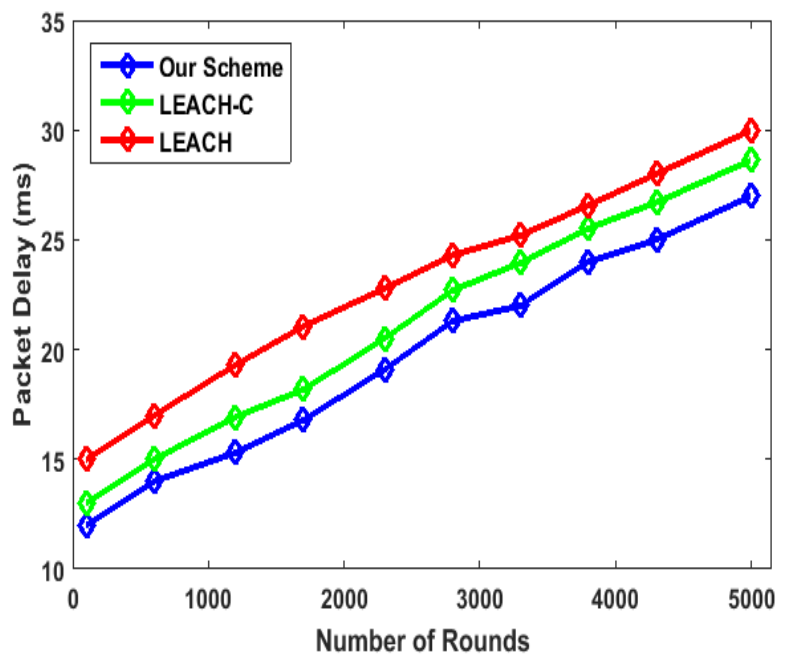

Fig. 5. Packet delay received

Fig. 6 reflects the communication overhead caused due to data transmission between the BS and nodes. Our scheme outperforms the other two schemes by minimizing the number of communications between BS and nodes. In LEACH, most of the nodes communicate their data directly towards the sink that not only increases communication overhead but also affects the lifespan of network by dissipating more energy during these communications. However, LEACH-C and our proposed scheme do minimal communication. Thus, energy is saved for information gathering that results in improved lifespans of the participating nodes.

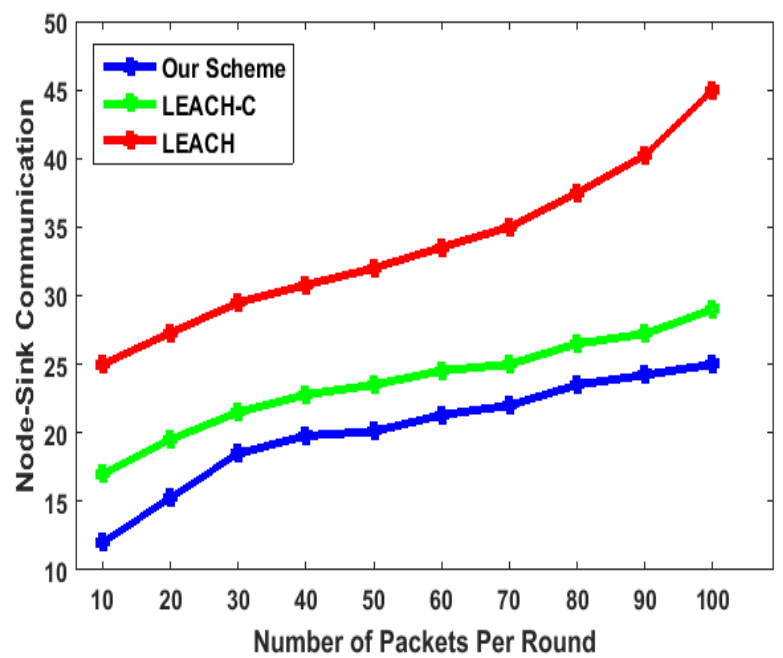

Fig. 6. Node-Sink communication overhead

\section{Conclusion}

Energy efficiency is a very important aspect of the wireless sensor networks during the aggregation procedure. In this work, we have proposed a novel energy efficient mechanism that introduces a super node for data aggregation. It collects the information from $\mathrm{CHs}$ and aggregates to eliminates existing any data redundancy before transmitting towards sink. This not only saves nodes energy but also reduces the number of communications in WSN. We have presented some preliminary evaluations of the proposed protocol where results are encouraging in terms of communication overhead, lifespan and energy consumption when compared with some of the existing protocols. However, more work needs to be done for packet latency that we intend to perform in the future.

\section{REFERENCES}

[1] J. N. Al-Karaki and A. E. Kamal, "Routing techniques in wireless sensor networks: a survey," IEEE Wireless Communications, vol. 11, no. 6, pp. 6-28, Dec 2004.

[2] J. Mousset, H. Zhou, and K. Hou, "One-hop broadcast routing protocol for wireless sensor," 2011 First International Conference on Instrumentation, Measurement, Computer, Communication and Control, pp. 804807, Oct 2011.

[3] W. B. Heinzelman, "Application-specific protocol architectures for wireless networks," Ph.D. dissertation, Cambridge, MA, USA, 2000.

[4] L. Ya, W. Pengjun, L. Rong, Y. Huazhong, and L. Wei, "Reliable energyaware routing protocol for heterogeneous wsn based on beaconing," 16th International Conference on Advanced Communication Technology, pp. 109-112, Feb 2014.

[5] Y. Li, A. Zhang, and Y. Liang, "Improvement of leach protocol for wireless sensor networks," 2013 Third International Conference on Instrumentation, Measurement, Computer, Communication and Control, pp. 322-326, Sept 2013.

[6] R. K. Kodali and N. Sarma, "Energy efficient routing protocols for wsn's," 2013 International Conference on Computer Communication and Informatics, pp. 1-4, Jan 2013. 
[7] V. Gupta and R. Pandey, "Research on energy balance in hierarchical clustering protocol architecture for wsn," 2014 International Conference on Parallel, Distributed and Grid Computing, pp. 115-119, Dec 2014.

[8] B. Xi-rong, Q. Zhi-tao, Z. Xue-feng, and Z. Shi, "An efficient energy cluster-based routing protocol for wireless sensor networks," 2009 Chinese Control and Decision Conference, pp. 4716-4721, June 2009.

[9] P. Xue-feng and L. La-yuan, "Design of an energy balanced based routing protocol for wsn," 2011 6th IEEE Joint International Information Technology and Artificial Intelligence Conference, vol. 2, pp. 366-369, Aug 2011.

[10] A. Yan and B. Wang, "An adaptive wsn clustering scheme based on neighborhood energy level," 2017 IEEE 3rd Information Technology and Mechatronics Engineering Conference (ITOEC), pp. 1170-1173, Oct 2017.

[11] A. Biazi, C. Marcon, F. Shubeita, L. Poehls, T. Webber, and F. Vargas,
"A dynamic tdma-based sleep scheduling to minimize wsn energy consumption," 2016 IEEE 13th International Conference on Networking, Sensing, and Control (ICNSC), pp. 1-6, April 2016.

[12] A. Liu, J. Ren, X. Li, Z. Chen, and X. S. Shen", “"design principles and improvement of cost function based energy aware routing algorithms for wireless sensor networks"," "Computer Networks", vol. 56, no. 7, pp. 1951 - 1967, 2012.

[13] W. Han, Y. Zhang, X. Wang, J. Li, M. Sheng, and X. Ma, "Orthogonal power division multiple access: A green communication perspective," IEEE Journal on Selected Areas in Communications, vol. 34, no. 12, pp. 3828-3842, Dec 2016.

[14] M. L. Rajaram, E. Kougianos, S. P. Mohanty, and U. Choppali, "Wireless sensor network simulation frameworks: A tutorial review: Matlab/simulink bests the rest," IEEE Consumer Electronics Magazine, vol. 5, no. 2, pp. 63-69, 2016. 